\title{
Research Article \\ Total Ponderomotive Force on an Extended Test Body
}

\author{
D. Langemann \\ Institute of Computational Mathematics, Technical University of Braunschweig, Pockelsstr. 14, \\ 38106 Braunschweig, Germany \\ Correspondence should be addressed to D. Langemann, d.langemann@tu-bs.de
}

Received 3 July 2009; Accepted 27 August 2009

Recommended by Vladislav Rustemovich Khalilov

Droplets on insulating material suffer a nonvanishing total ponderomotive force because of the inhomogeneity of the surrounding electric field. A series expansion of this total force is proven in a two-dimensional setting by determining the line charge density at the boundary of the test body via a Fredholm integral equation, which is solved by Fourier techniques. The influence of electric charges in the neighborhood of the test body can be estimated as well as the convergence speed of the series expansion. In all realistic applications the series converges very fast. The numerical effort in the simulation of the motion of rainwater droplets on outdoor insulators reduces considerably.

Copyright (C) 2009 D. Langemann. This is an open access article distributed under the Creative Commons Attribution License, which permits unrestricted use, distribution, and reproduction in any medium, provided the original work is properly cited.

\section{Introduction}

The total force $\mathbf{F}$ acting on an uncharged test body in an inhomogeneous electric field is called ponderomotive force [1]. It is the reason why an uncharged droplet moves within an electric field in experiments [2], and it is an important influence factor for the motion of realistic rainwater droplets on outdoor high-voltage insulating equipment $[3,4]$.

The simulation of moving rainwater droplets requires the determination of the ponderomotive force, and the concentration on the total force $\mathbf{F}$ is a reasonable simplification with respect to other influences like the weather. The computation of the electric fieldaround the droplet is a computationally expensive task, which is dealt with finite integration techniques in $[5,6]$ or by finite elements on an adaptive triangular grid in [4]. Similarly, a related problem is solved in [7] in the investigation of ferromagnetic fluids.

In plasma physics, the ponderomotive force plays an important role [8], and therefore the ponderomotive force sometimes seems to be strictly related to oscillating electric fields. However, the same inhomogeneity of the electric field causes a nonvanishing divergence of the Maxwell stress tensor and thus a force in classical electrodynamics as well as in plasma physics. 
In a previous work, the droplet was idealized to a round conductive and undeformable test body, and an explicit expression of the total force $\mathbf{F}$ could be derived [9-11]. This explicit expression is a fast-converging series expansion in inhomogeneity indicators $\mathbf{I}_{k}^{(\Psi)}$, which are computed in terms of the undisturbed potential $\Psi$ in the absence of the test body.

Until now, the series expansion was proven by introducing an auxiliary domain $\Theta$ containing the domain $\Omega$ of the round test body. The potential $\Psi$ at the boundary $\partial \Theta$ of the auxiliary domain was used as Dirichlet boundary condition for the potential $\Phi$ disturbed by the presence of the test body. After having derived the series expansion of $\mathbf{F}$ in a fixed auxiliary domain $\Theta$, an argumentation for extending the domain $\Theta$ was used.

This procedure is insatisfactory, because boundary conditions of $\Psi$ and additional charges in the vicinity of the test body are not regarded, and they would let fail the argument of an extending $\Theta$.

Here, we give a new proof of the series expansion in inhomogeneity indicators. This new proof concentrates on the line charge $\sigma_{\Gamma}$ at the boundary $\Gamma=\partial \Omega$ of the test body, and it does not need any auxiliary domain. Basing on the proof, a new estimation of the damped influence of neighbored charges on the test body is given. By similar investigations, the diminishing behavior of the terms in the series for $\mathbf{F}$ is estimated. It can be shown that the series converges at least as fast as a geometric series.

The present paper is organized as follows. It starts with preliminaries and notations where the undisturbed electric potential $\Psi$ and the potential $\Phi$ disturbed by the round, conductive, and charge-free test body are presented. Here, the generated line charge density $\sigma_{\Gamma}$ at the boundary $\Gamma$ of the test body is introduced. Section 3 solves a Fredholm integral equation of first order by Fourier techniques and shows some facts which are needed to give the series expansion of $\mathbf{F}$ in inhomogeneity indicators. Then, Section 4 deals with the convergence behavior and the convergence speed of the series. Furthermore, it discusses the diminishing speed of the summands in the series expansion and the decreasing influence of more remote electric charges.

The paper ends with a short conclusion resuming the results and giving an outlook to further investigations like, for example, the analogous investigation in higher dimensions or the extension of the results to more general shapes of the test body.

\section{Preliminaries and Notations}

We denote the points of the two-dimensional Euclidean space $\mathbb{R}^{2}$ by $\mathbf{x}=\left(x_{1}, x_{2}\right)^{\mathrm{T}}$. The polar co-ordinates are named $\mathbf{x}=\mathbf{x}(r, \varphi)$. The two-dimensional Laplacian operator $-\Delta: \mathrm{H}^{1}\left(\mathbb{R}^{2}\right) \rightarrow$ $\mathrm{H}^{-1}\left(\mathbb{R}^{2}\right)$ maps $\mathrm{H}^{-1}$-functions in a weak or distributional sense.

The undisturbed electric potential $\Psi \in \mathrm{H}^{1}\left(\mathbb{R}^{2}\right)$ is generated by a charge density $\rho \in \mathrm{H}^{-1}\left(\mathbb{R}^{2}\right)$. The dielectricity constant is denoted by $\varepsilon_{0}$. We normalize any possible relative dielectricity, and the potential equation is

$$
\begin{gathered}
-\varepsilon_{0} \Delta \Psi(\mathbf{x})=\rho(\mathbf{x}) \quad \text { for } \mathbf{x} \in \mathbb{R}^{2}, \\
\lim _{\|\mathbf{x}\| \rightarrow \infty} \Psi(\mathbf{x})=0,
\end{gathered}
$$

where the boundary condition at infinity assures uniqueness of the solution [12]. It means that the potential tends to zero for any unbounded sequence of points in $\mathbb{R}^{2}$. In two dimensions, that is, in $\mathbb{R}^{2}$, the boundary condition at infinity contains the realistic condition 
that the sum of all charges in a bounded domain tends to zeros if the domain is increased onto the whole space. This is an artefact of the two-dimensional setting. In the natural case of three dimensions, it is not necessary to require a vanishing sum of the electric charge [13].

The formulation (2.1) includes possible boundary conditions at bounded domains because they are effected by suitable charge densities too. The fundamental solution $g$ of the two-dimensional Laplacian $-\Delta$ is

$$
g(\mathbf{z})=-\frac{1}{2 \pi} \ln \|\mathbf{z}\|
$$

and Green's formula for (2.1) reads

$$
\Psi(\mathbf{x})=\frac{1}{\varepsilon_{0}} \int_{\mathbb{R}^{2}} \rho(\mathbf{y}) g(\mathbf{x}-\mathbf{y}) \mathrm{d} \mathbf{y},
$$

where dy denotes an area element here.

The test body occupies the round domain $\Omega=\left\{\mathbf{x}:\|\mathbf{x}\|<r_{\Gamma}\right\}$ with radius $r_{\Gamma}$, where $\|\cdot\|$ is the Euclidean norm. The boundary of $\Omega$ is named $\Gamma=\partial \Omega=\left\{\mathbf{x}:\|\mathbf{x}\|=r_{\Gamma}\right\}$. The outer normal is denoted by $\mathbf{n}=\mathbf{x} / r_{\Gamma}$ for $\mathbf{x} \in \Gamma$. We denote the projection of $\mathbf{x} \in \mathbb{R}^{2} \backslash\{\mathbf{0}\}$ onto the boundary $\Gamma$ as $\mathbf{x}_{\Gamma}=r_{\Gamma} \mathbf{x} /\|\mathbf{x}\|$.

The test body influences the undisturbed potential, and the resulting potential is called the potential $\Phi \in \mathrm{H}^{1}\left(\mathbb{R}^{2}\right)$ disturbed by the presence of the test body. Since the test body is conductive, the potential $\Phi$ is constant in $\Omega$. We denote $\Phi(\mathbf{x})=c$ for $\mathbf{x} \in \bar{\Omega}$, where the constant $c$ is unknown until now. Furthermore outside $\Omega$, it is generated by the charge density $\rho$, too. The conductivity condition of the test body requires

$$
\bar{\Omega} \cap \operatorname{supp} \rho=\emptyset .
$$

The potential $\Phi$ bends on $\Gamma$, and thus it generates an additional charge density $\sigma \in \mathrm{H}^{-1}\left(\mathbb{R}^{2}\right)$ concentrated at $\Gamma$ due to the separation of charges there, which obeys $\sigma(\mathbf{x})=-\varepsilon_{0} \Delta \Phi(\mathbf{x})$ for $\mathbf{x} \in \Gamma$ in a weak sense and is identical to zero elsewhere. Since $\sigma$ is concentrated on $\Gamma$, it can be expressed as the product

$$
\sigma(\mathbf{x})=\sigma_{\Gamma}\left(\mathbf{x}_{\Gamma}\right) \delta\left(\|\mathbf{x}\|-r_{\Gamma}\right)
$$

of the line charge density $\sigma_{\Gamma} \in \mathrm{C}(\Gamma)$ and the one-dimensional Dirac distribution $\delta$. We show a lemma about the line density.

Lemma 2.1. The generated line charge density is $\sigma_{\Gamma}\left(\mathbf{x}_{\Gamma}\right)=-\varepsilon_{0} \nabla \Phi\left(\mathbf{x}_{\Gamma}\right) \mathbf{n}$ with the unilateral outer gradient $\nabla \Phi\left(\mathbf{x}_{\Gamma}\right)$ at $\mathbf{x}_{\Gamma} \in \Gamma$.

Proof. We denote $\sigma(\mathbf{x})=\sigma(r, \varphi)$ in polar coordinates, $\Phi(\mathbf{x})=\Phi(r, \varphi)$ and $\sigma_{\Gamma}\left(\mathbf{x}_{\Gamma}\right)=\sigma_{\Gamma}(\varphi)$, respectively. With $\alpha>0$, (2.5) gives

$$
\sigma_{\Gamma}\left(\mathbf{x}_{\Gamma}\right)=\int_{r_{\Gamma}-\alpha}^{r_{\Gamma}+\alpha} \sigma(r, \varphi) \mathrm{dr}=\lim _{\alpha \rightarrow 0^{+}} \int_{r_{\Gamma}-\alpha}^{r_{\Gamma}+\alpha} \sigma(r, \varphi) \mathrm{dr}
$$


The Laplacian of the disturbed potential $\Phi$ is

$$
\Delta \Phi(\mathbf{x})=\left[\frac{\partial^{2}}{\partial r^{2}}+\frac{1}{r} \frac{\partial}{\partial r}+\frac{1}{r^{2}} \frac{\partial^{2}}{\partial \varphi^{2}}\right] \Phi(r, \varphi)
$$

in polar coordinates. With the Heaviside function $H$, the Laplacian for the potential $\Phi$ bending at $\Gamma$ reads

$$
\Delta \Phi(r, \varphi)=\delta\left(r-r_{\Gamma}\right)\left[\frac{\partial}{\partial r_{+}}-\frac{\partial}{\partial r_{-}}\right] \Phi(r, \varphi)+H\left(r-r_{\Gamma}\right)\left[\frac{\partial^{2}}{\partial r_{+}^{2}}+\frac{1}{r} \frac{\partial}{\partial r_{+}}\right] \Phi(r, \varphi)+\frac{1}{r^{2}} \frac{\partial^{2}}{\partial \varphi^{2}} \Phi(r, \varphi),
$$

where the unilateral derivatives are marked by the indices + and - , respectively. Since $\Phi$ is constant on $\Gamma$ and thus independent of the angle $\varphi,(2.6)$ together with the constance of $\Phi$ inside $\Omega$ gives

$$
\sigma_{\Gamma}\left(\mathbf{x}_{\Gamma}\right)=-\varepsilon_{0} \frac{\partial}{\partial r^{+}} \Phi\left(r_{\Gamma}, \varphi\right)=-\varepsilon_{0} \frac{\partial \Phi\left(\mathbf{x}_{\Gamma}\right)}{\partial \mathbf{n}}
$$

because $\Phi$ is constant in the orthogonal, angular direction.

Since the test body is free of charge, the integral over $\sigma$ and hence over $\sigma_{\Gamma}$ vanishes, and the potential equation for the disturbed potential $\Phi$ reads

$$
\begin{gathered}
-\varepsilon_{0} \Delta \Phi(\mathbf{x})=\rho(\mathbf{x}) \quad \text { for } \mathbf{x} \in \mathbb{R}^{2} \backslash \bar{\Omega} \\
\Phi(\mathbf{x})=c \quad \text { for } \mathbf{x} \in \bar{\Omega} \\
\int_{\Gamma} \nabla \Phi(\mathbf{x}) \mathbf{n} \mathrm{d} \mathbf{x}=0 \\
\lim _{\|\mathbf{x}\| \rightarrow \infty} \Phi(\mathbf{x})=0
\end{gathered}
$$

where the first equation is Poisson's equation with the charge density $\rho$ outside the test body. The second equation encodes the conductivity of the test body and, therefore, the constance of the potential $\Phi$ in the test body and particularly at its boundary $\Gamma$. The third relation in (2.10) contains the fact that the test body is free of charge, (cf. Lemma 2.1). Again, the boundary condition of $\Phi$ at infinity assures uniqueness. The line element on $\Gamma$ is denoted by $\mathrm{d} \mathbf{x}$ too. The constant $c$ is determined by the charge-free condition in $(2.10)[9,10]$.

Finally, the total ponderomotive force is given by

$$
\mathbf{F}=\frac{\varepsilon_{0}}{2} \int_{\Gamma}|\nabla \Phi(\mathbf{x})|^{2} \mathbf{n} \mathrm{d} \mathbf{x}=\frac{1}{2} \int_{\Gamma} \sigma_{\Gamma}\left(\mathbf{x}_{\Gamma}\right)\left|\nabla \Phi\left(\mathbf{x}_{\Gamma}\right)\right| \mathbf{n} \mathrm{d} \mathbf{x}_{\Gamma}=\frac{1}{2 \varepsilon_{0}} \int_{\Gamma} \sigma_{\Gamma}\left(\mathbf{x}_{\Gamma}\right)^{2} \mathbf{n} \mathrm{d} \mathbf{x}_{\Gamma},
$$

where the second equivalence follows from Lemma 2.1. The existence of the integral in the defining (2.11) is not immediately obvious because the trace theorem [13] assures only 
$\left.\nabla \Phi\right|_{\Gamma} \in \mathrm{H}^{-1 / 2}(\Gamma)$ in general. However, due to the smooth boundary $\Gamma$, it holds true that $\left.\nabla \Phi\right|_{\Gamma} \in \mathrm{L}_{2}(\Gamma)[14]$, and the integral in (2.11) is meaningful.

Now, the disturbed potential $\Phi$ is determined by the charge density $\rho$ and by the generated charge density $\sigma$. Thus, Green's formula reads

$$
\Phi(\mathbf{x})=\frac{1}{\varepsilon_{0}} \int_{\mathbb{R}^{2}}[\rho(\mathbf{y})+\sigma(\mathbf{y})] g(\mathbf{x}-\mathbf{y}) \mathrm{d} \mathbf{y}=\Psi(\mathbf{x})+\frac{1}{\varepsilon_{0}} \int_{\Gamma} \sigma_{\Gamma}(\mathbf{y}) g(\mathbf{x}-\mathbf{y}) \mathrm{d} \mathbf{y},
$$

where $\mathrm{dy}$ in the first term denotes an area element, and in the second term, it denotes the line element on $\Gamma$, respectively, to the integration domain.

By the way, (2.12) includes the known fact that the influence of the test body onto the neighborhood diminishes at least with the decreasing behavior of the fundamental solution. Since the test body is charge-free and the sum of the line charge $\sigma_{\Gamma}$ is vanishing, the influence actually diminishes like the reciprocal of the distance in the two-dimensional setting.

\section{Line Charge Density and a Fredholm Integral Equation}

We use the preliminaries for deriving a Fredholm integral equation for the line charge density. Further, we will solve it in dependence of the undisturbed potential $\Psi$. This solution will enable us to express the total ponderomotive force $\mathbf{F}$ in terms of $\Psi$.

Theorem 3.1. Let $r_{\Gamma} \in(0,1)$. If the line charge density $\sigma_{\Gamma}$ fulfills

$$
\int_{\Gamma} \sigma_{\Gamma}(\mathbf{y}) g(\mathbf{x}-\mathbf{y}) \mathrm{d} \mathbf{y}=\frac{\varepsilon_{0}}{2 \pi r_{\Gamma}} \int_{\Gamma} \Psi(\mathbf{y}) \mathrm{d} \mathbf{y}-\varepsilon_{0} \Psi(\mathbf{x})
$$

for all $\mathbf{x} \in \Gamma$, then $\sigma_{\Gamma}$ generates $\Phi$ with $\int_{\Gamma} \nabla \Phi(\mathbf{x}) \mathbf{n d} \mathbf{x}=0$ and with $\Phi(\mathbf{x})=c$ for all $\mathbf{x} \in \bar{\Omega}$.

Proof. Starting with a vanishing difference and using condition (3.1), it holds true that

$$
0=\int_{\Gamma}\left(\frac{\varepsilon_{0}}{2 \pi r_{\Gamma}} \int_{\Gamma} \Psi(\mathbf{y}) \mathrm{d} \mathbf{y}-\varepsilon_{0} \Psi(\mathbf{x})\right) \mathrm{d} \mathbf{x}=\int_{\Gamma} \int_{\Gamma} \sigma_{\Gamma}(\mathbf{y}) g(\mathbf{x}-\mathbf{y}) \mathrm{d} \mathbf{y d} \mathbf{x}
$$

Hence, one finds after changing the integration order

$$
0=\int_{\Gamma} \sigma_{\Gamma}(\mathbf{y}) \int_{\Gamma} g(\mathbf{x}-\mathbf{y}) \mathrm{d} \mathbf{x} \mathrm{d} \mathbf{y}=C \int_{\Gamma} \sigma_{\Gamma}(\mathbf{y}) \mathrm{d} \mathbf{y}
$$

with $C=\int_{\Gamma} g(\mathbf{x}-\mathbf{y}) \mathrm{d} \mathbf{x}$, which is independent of $\mathbf{y}$ because of the rotational symmetry of $\Gamma$. With $\left\|\mathbf{x}\left(r_{\Gamma}, \varphi\right)-\mathbf{y}\left(r_{\Gamma}, 0\right)\right\|=2 r_{\Gamma} \sin (\varphi / 2)$, the constant $C$ is calculated by

$$
C=-\frac{1}{2 \pi} \int_{0}^{2 \pi} \ln \left(2 r_{\Gamma} \sin \frac{\varphi}{2}\right) r_{\Gamma} \mathrm{d} \varphi=-r_{\Gamma} \ln r_{\Gamma}
$$


Hence, $r_{\Gamma} \in(0,1)$ implies $C \neq 0$, and (3.3) together with Lemma 2.1 is the first proposition. Again the requirement $r_{\Gamma}<1$ is an artefact of the two-dimensional setting, which does not occur in three dimensions because the fundamental solution does not have any zeros then.

Next, (2.12) with the condition (3.1) gives

$$
\Phi(\mathbf{x})=\frac{1}{2 \pi r_{\Gamma}} \int_{\Gamma} \Psi(\mathbf{y}) \mathrm{d} \mathbf{y}=c \quad \text { for all } \mathbf{x} \in \Gamma
$$

which is independent of $\mathbf{x}$. Since $\Phi$ is constant on $\Gamma$ and since there is no electric charge in $\Omega$ (cf. (2.4)) it is true that $\Phi$ is constant in $\Omega$.

Let us remark that the restriction $r_{\Gamma}<1$ does not occur in higher dimension because the fundamental solutions do not change sign then. However, even $r_{\Gamma}<1$ can be overcome by the transformation of coordinates.

Corollary 3.2. It holds true that $c=\Psi(0)$ for every undisturbed potential $\Psi$.

Proof. The undisturbed potential $\Psi$ is a potential function in $\bar{\Omega}$ because of (2.4), and (3.5) yields the proposition.

Using the proof of Corollary 3.2, condition (3.1) reads

$$
\int_{\Gamma} \sigma_{\Gamma}(\mathbf{y}) g(\mathbf{x}-\mathbf{y}) \mathrm{d} \mathbf{y}=\varepsilon_{0}[\Psi(\mathbf{0})-\Psi(\mathbf{x})]
$$

for all $\mathbf{x} \in \Gamma$. This is a Fredholm integral equation of first order [15] for the determination of $\sigma_{\Gamma}$. In the following, the integral equation (3.6) or (3.1), respectively, is solved by Fourier techniques with the aim to determine $\sigma_{\Gamma}$ and the total ponderomotive force $\mathbf{F}$ in (2.11).

Since the domain $\bar{\Omega}$ is free of charge (cf. (2.4)) $\Psi$ is a potential function, and it can be given as

$$
\Psi(\mathbf{x})=\Psi(r, \varphi)=\sum_{k=-\infty}^{\infty} \tilde{a}_{k} r^{|k|} \mathrm{e}^{\mathrm{i} k \varphi}
$$

for all $\mathbf{x} \in \bar{\Omega}$, that is, for $r \in\left[0, r_{\Gamma}\right]$. The notation $a_{k}=\tilde{a}_{k} r_{\Gamma}^{|k|}, k \in \mathbb{Z}$ gives

$$
\Psi\left(r_{\Gamma}, \varphi\right)=\sum_{k=-\infty}^{\infty} a_{k} \mathrm{e}^{\mathrm{i} k \varphi}
$$

In (3.8), we find the Fourier coefficients $a_{k}=c_{k}\left(\Psi\left(r_{\Gamma}, \cdot\right)\right)$ which are defined by

$$
c_{k}(f)=\frac{1}{2 \pi} \int_{0}^{2 \pi} f(\varphi) \mathrm{e}^{-\mathrm{i} k \varphi} \mathrm{d} \varphi
$$


for a $2 \pi$-periodic function $f$. We identify the point $\mathbf{x}_{\Gamma}=\mathbf{x}_{\Gamma}\left(r_{\Gamma}, \varphi\right)$ with the angle $\varphi$ of the polar coordinates, and (3.6) reads

$$
\int_{0}^{2 \pi} \sigma_{\Gamma}(\psi) g(|\varphi-\psi|) r_{\Gamma} \mathrm{d} \psi=\varepsilon_{0}\left[\Psi(\mathbf{0})-\Psi\left(r_{\Gamma}, \varphi\right)\right]=-\varepsilon_{0} \sum_{k=-\infty, k \neq 0}^{\infty} a_{k} \mathrm{e}^{\mathrm{i} k \varphi}
$$

for all $\varphi \in[0,2 \pi)$ and with $g=g(\eta)=\ln \left(2 r_{\Gamma} \sin (\eta / 2)\right)$ for $\eta \in[0,2 \pi]$ like in (3.4).

Since (3.10) is a convolution of the $2 \pi$-periodic functions $\sigma_{\Gamma}=\sigma_{\Gamma}(\psi)$ and $g=g(\eta)$, it holds true [16] that

$$
2 \pi c_{k}\left(\sigma_{\Gamma}\right) c_{k}(g)=c_{k}\left(\sigma_{\Gamma} \circ g\right)=-\frac{\varepsilon_{0} a_{k}}{r_{\Gamma}}
$$

for $k \neq 0$ and $c_{0}\left(\sigma_{\Gamma}\right) c_{0}(g)=0$. We compute $c_{k}(g)=1 /(4 \pi|k|)$ for $k \neq 0$, and we get

$$
c_{k}\left(\sigma_{\Gamma}\right)=-\frac{2 \varepsilon_{0}|k|}{r_{\Gamma}} a_{k}
$$

for $k \neq 0$. The term $c_{0}\left(\sigma_{\Gamma}\right)$ is not determined by the integral equations (3.6) or (3.10), respectively (cf. Fredholm's alternative [15]). However, (2.10) yields $c_{0}\left(\sigma_{\Gamma}\right)=0$ in the chargefree condition. Therefore, we find

$$
\sigma_{\Gamma}(\varphi)=-\frac{2 \varepsilon_{0}}{r_{\Gamma}} \sum_{k=-\infty}^{\infty}|k| a_{k} \mathrm{e}^{\mathrm{i} k \varphi}
$$

Finally, the total ponderomotive force in $(2.11)$ is

$$
\mathbf{F}=\frac{2 \varepsilon_{0}}{r_{\Gamma}^{2}} \int_{0}^{2 \pi}\left(\sum_{k=-\infty}^{\infty}|k| a_{k} \mathrm{e}^{\mathrm{i} k \varphi}\right)^{2} \mathbf{n} r_{\Gamma} \mathrm{d} \varphi \text { with } \mathbf{n}=\left(\begin{array}{c}
\cos \varphi \\
\sin \varphi
\end{array}\right)
$$

In fact (3.14) is already an expression for $\mathbf{F}$ in terms of the undisturbed potential $\Psi$, in particular, in the Fourier coefficents of its restriction to the boundary of the round test body. In the following, we develop a more convenient expression in terms of $\Psi$ at the origin $\mathbf{0 .}$

We consider the components of the force $\mathbf{F}=\left(F_{1}, F_{2}\right)$, and we have

$$
F_{1}=\frac{2 \varepsilon_{0}}{r_{\Gamma}^{2}} \sum_{k=-\infty}^{\infty} \sum_{\ell=-\infty}^{\infty}|k||\ell| a_{k} a_{-\ell} \int_{0}^{2 \pi} \frac{\mathrm{e}^{\mathrm{i} \varphi}+\mathrm{e}^{-\mathrm{i} \varphi}}{2} \mathrm{e}^{\mathrm{i}(k-\ell) \varphi} r_{\Gamma} \mathrm{d} \varphi
$$

The evaluation of the integral gives

$$
F_{1}=\frac{2 \varepsilon_{0} \pi}{r_{\Gamma}} \sum_{k=-\infty}^{\infty} \sum_{\ell=-\infty}^{\infty}|k||\ell| a_{k} a_{-\ell}\left(\delta_{k, \ell+1}+\delta_{k, \ell-1}\right)
$$


with the Kronecker symbol $\delta_{k \ell}$, which is $\delta_{k \ell}=1$ if and only if $k=\ell$ and vanishing else. Since $\Psi\left(r_{\Gamma}, \cdot\right)$ is real-valued, it holds true $\bar{a}_{-k}=a_{k}$, and the double sum reduces to

$$
F_{1}=\frac{4 \pi \varepsilon_{0}}{r_{\Gamma}} \sum_{k=1}^{\infty} k(k+1)\left(\bar{a}_{k} a_{k+1}+a_{k} \bar{a}_{k+1}\right)
$$

Analogously, we find

$$
F_{2}=\frac{4 \pi \mathrm{i} \varepsilon_{0}}{r_{\Gamma}} \sum_{k=1}^{\infty} k(k+1)\left(\bar{a}_{k} a_{k+1}-a_{k} \bar{a}_{k+1}\right)
$$

On the other hand, the formula of Moivre gives (3.7) in Cartesian co-ordinates as

$$
\Psi(\mathbf{x})=\Psi\left(x_{1}, x_{2}\right)=\tilde{a}_{0}+\sum_{k=1}^{\infty} \tilde{a}_{k}\left(x_{1}+\mathrm{i} x_{2}\right)^{k}+\sum_{k=1}^{\infty} \tilde{a}_{-k}\left(x_{1}-\mathrm{i} x_{2}\right)^{k}
$$

As defined in $[9,10]$ the inhomogeneity indicators are

$$
\mathbf{I}_{k}^{(\Psi)}(\mathbf{x})=\nabla\left[\nabla^{k} \Psi(\mathbf{x}): \nabla^{k} \Psi(\mathbf{x})\right]
$$

where ":" denotes the full tensor contraction of the $k$ th derivatives $\nabla^{k} \Psi$. The inhomogeneity indicators encode the deviation of the undisturbed potential $\Psi$ from the potential of a homogeneous electric field, which has vanishing inhomogeneity indicators. In [9], it is shown that $a_{k}=\tilde{a}_{k} r_{\Gamma}^{|k|}$ in (3.19) implies

$$
\mathbf{I}_{k}^{(\Psi)}(\mathbf{0})=\frac{2^{k+1} k !(k+1) !}{r_{\Gamma}^{2 k+1}}\left(\begin{array}{c}
\bar{a}_{k} a_{k+1}+a_{k} \bar{a}_{k+1} \\
\mathrm{i} \bar{a}_{k} a_{k+1}-\mathrm{i} a_{k} \bar{a}_{k+1}
\end{array}\right)
$$

The comparison of this result with (3.17) and (3.18) yields the series

$$
\mathbf{F}=2 \pi \varepsilon_{0} \sum_{k=1}^{\infty} \frac{k}{k !^{2}} \frac{r_{\Gamma}^{2 k}}{2^{k}} \mathbf{I}_{k}^{(\Psi)}(\mathbf{0}),
$$

which is the proposed relation between the derivatives of the undisturbed potential $\Psi$ at the middle of the test body, which was set to 0 without loss of generality here. Equation (3.22) allows us to separate the computation of the electric potential $\Psi$ and the determination of the total ponderomotive force $\mathbf{F}$ on an uncharged conductive body. So, the motion of the body inside the electric field can be determined with a single computation of the undisturbed electric potential. The following section will answer the question of the convergence speed of the series (3.22). 


\section{Convergence Speed of the Series}

The domain of the test body itself is free of charge. The charge density $\rho$ has a support which is strictly remote of the test body (cf. (2.4)).

We investigate how the terms of the series (3.22) or rather (3.17) and (3.18), respectively, depend on the charge density $\rho$. We remark that the undisturbed potential $\Psi$ depends linearly on $\rho$ in (2.3), and hence the coefficients $a_{k}$ in (3.8) do so. Finally, the line charge $\sigma_{\Gamma}$ depends linearly on $\rho$ via (3.13).

From a physical viewpoint, it is obvious - and we see it in the formulas too - that the influence of the charges on the total force diminishes with the distance of the charge from the test body. So, we will start with the investigation of a single point charge in the distance $t>r_{\Gamma}$ from the origin $\mathbf{0}$. Since this setting is rotationally symmetric, this means, for example, $\rho(r, \varphi)=\delta(t-r) \delta(\varphi)$.

The undisturbed electric potential generated by this single point charge is

$$
\Psi(r, \varphi)=-\frac{1}{2 \pi \varepsilon_{0}} \ln \sqrt{t^{2}+r^{2}-2 r t \cos \varphi}
$$

with the Fourier coefficients

$$
a_{k}=c_{k}\left(\Psi\left(r_{\Gamma}, \cdot\right)\right)=-\frac{1}{(2 \pi)^{2} \varepsilon_{0}} \int_{0}^{2 \pi} \mathrm{e}^{-\mathrm{i} k \varphi} \ln \sqrt{t^{2}+r_{\Gamma}^{2}-2 r_{\Gamma} t \cos \varphi} \mathrm{d} \varphi
$$

as in (3.8) at the boundary $\Gamma$ of the test body. With this abbreviation $\tau=t r_{\Gamma} /\left(t^{2}+r_{\Gamma}^{2}\right)<1 / 2$ holds true, that is

$$
\ln \sqrt{t^{2}+r_{\Gamma}^{2}-2 r_{\Gamma} t \cos \varphi}=\frac{1}{2} \ln \left(t^{2}+r_{\Gamma}^{2}\right)+\frac{1}{2} \ln (1-2 \tau \cos \varphi) .
$$

Thus in (4.2), the Fourier coefficients with $k>0$ are

$$
a_{k}=-\frac{1}{8 \pi^{2} \varepsilon_{0}} \int_{0}^{2 \pi} \mathrm{e}^{-\mathrm{i} k \varphi} \ln (1-2 \tau \cos \varphi) \mathrm{d} \varphi=\frac{\mathrm{i}}{4 k \pi^{2} \varepsilon_{0}} \int_{0}^{2 \pi} \mathrm{e}^{-\mathrm{i} k \varphi} \frac{\tau \sin \varphi}{1-2 \tau \cos \varphi} \mathrm{d} \varphi .
$$

Since $|2 \tau \cos \varphi|<1$, the term $(1-2 \tau \cos \varphi)^{-1}$ can be written as geometric series, and we find the relation

$$
a_{k}=\frac{\tau}{8 k \pi^{2} \varepsilon_{0}} \int_{0}^{2 \pi}\left(\mathrm{e}^{-\mathrm{i}(k-1) \varphi}-\mathrm{e}^{-\mathrm{i}(k+1) \varphi}\right) \sum_{j=0}^{\infty}\left[\tau\left(\mathrm{e}^{\mathrm{i} \varphi}+\mathrm{e}^{-\mathrm{i} \varphi}\right)\right]^{j} \mathrm{~d} \varphi .
$$

The binomials in the sum do not vanish only in the cases that the exponents coincide with the exponents $\mathrm{i}(k-1) \varphi$ and $-\mathrm{i}(k+1) \varphi$ in the sinus-term. Hence, we get

$$
a_{k}=\frac{\tau^{k+1}}{4 k \pi \varepsilon_{0}}\left[\sum_{n=0}^{\infty}\left(\begin{array}{c}
2 n-1+k \\
n
\end{array}\right) \tau^{2 n-1}-\sum_{n=0}^{\infty}\left(\begin{array}{c}
2 n+1+k \\
n
\end{array}\right) \tau^{2 n+1}\right] .
$$


After separation of the first summand for $n=0$ and an index shift in the first sum, we find

$$
a_{k}=\frac{1}{4 \pi \varepsilon_{0}}\left[\frac{\tau^{k}}{k}+\tau^{k+2} A(k, \tau)\right] \text { with } A(k, \tau)=\sum_{n=0}^{\infty} \frac{\tau^{2 n}}{n+1}\left(\begin{array}{c}
2 n+1+k \\
n
\end{array}\right) .
$$

The hypergeometric expression $A(k, \tau)$ is monotonously increasing in $\tau$ for every $k>0$, and it holds true that

$$
A(k, 0)=1, \quad A\left(k, \frac{1}{2}\right)=\frac{4\left(2^{k}-1\right)}{k}
$$

However, (4.7) shows that the coefficients $a_{k}$ are positive for $k>0$, and (4.8) yields the estimation

$$
a_{k} \leq \frac{1}{4 k \pi \varepsilon_{0}}\left[\tau^{k}+4\left(2^{k}-1\right) \tau^{k+2}\right]<\frac{1}{4 k \pi \varepsilon_{0}}\left[\tau^{k}+(2 \tau)^{k+2}\right] .
$$

Finally, we see that the modulus of the series in (3.17) and (3.18)fulfils

$$
\left|F_{j}\right| \leq \frac{8 \pi \varepsilon_{0}}{r_{\Gamma}} \sum_{k=1}^{\infty} k(k+1) a_{k} a_{k+1}<\frac{1}{2 \pi \varepsilon_{0} r_{\Gamma}} \sum_{k=1}^{\infty}\left[\tau^{k}+(2 \tau)^{k+2}\right]\left[\tau^{k+1}+(2 \tau)^{k+3}\right]
$$

for $j \in\{1,2\}$, which leads to convergent geometric series because of $2 \tau<1$. After the evaluation of the geometric series in the right-hand expression in (4.10), we get the relation

$$
\left|F_{j}\right|<\frac{1}{2 \pi \varepsilon_{0} r_{\Gamma}}\left[\frac{\tau^{3}}{1-\tau^{2}}+\frac{24 \tau^{5}}{1-2 \tau^{2}}+\frac{32 \tau^{7}}{1-4 \tau^{2}}\right] \text {. }
$$

In realistic applications, electric charges are remote from the test body compared to the size of the test body, for example, droplets on insulating material, and thus often we have $\tau \ll 1 / 2$. Then, the series (3.17) and (3.18) and hence the series in (3.22) converges fast.

At the same time, (4.10) estimates the influence of remote charges to its neighborhood. For falling $\tau$, that is, for an increasing distance $t$ of the charge to the test body, it holds true that

$$
\left|F_{j}\right| \sim \mathcal{O}\left(\tau^{3}\right) \text { for } \tau \rightarrow 0 \text { with } \tau<\frac{r_{\Gamma}}{t}
$$

The discussion of this section is accomplished by the apprehensible fact that the influence of a charge distribution can be estimated by a concentrated absolute charge distribution at the nearest point of supp $\rho$ to the test body. By (4.7) we know that $\left|a_{k}\right| \leq q(t)$ with a positive and monotonously decreasing function $q$ in the case of a concentrated normed charge at distance $t$. Consequently, a distributed charge density gives

$$
\left|a_{k}\right| \leq\left|\int_{\operatorname{supp} \rho} \rho(\mathbf{x}) q(\|\mathbf{x}\|) \mathrm{d} \mathbf{x}\right| \leq \int_{\operatorname{supp} \rho}|\rho(\mathbf{x})| q(\|\mathbf{x}\|) \mathrm{d} \mathbf{x} \leq \min _{\mathbf{x} \in \operatorname{supp} \rho} q(\|\mathbf{x}\|) \int_{\operatorname{supp} \rho}|\rho(\mathbf{x})| \mathrm{d} \mathbf{x} .
$$


In fact (4.13) shows that the above investigation about the decreasing behavior of the summands in the series expansion in (3.22) are valid for a distributed charge density too. Furthermore, such a distributed charge density implies an even faster convergence, in particular, in the realistic case of a vanishing total charge.

\section{Conclusion}

We have developed a series expansion for the total ponderomotive force acting on a round, conductive, and charge-free test body in an homogeneous media. This is a reasonable approximation for rainwater droplets on insulating material in outdoor high-voltage equipment, because the total ponderomotive force only gives a tendency of their motion, which is additionally influenced by the weather, further external causes, and by the surface properties of the insulating material.

The motion of a rainwater droplet on insulating material [3-6] can be simulated by the determination of the time-dependent position of the test body, which moves under the influence of the ponderomotive force. Now, the series expansion in inhomogeneity indicators considerately reduces the numerical effort in this simulation. It requires only one solution of the field equation, and the derivatives needed in the determination of the inhomogeneity indicators can beread out for every position. Compared to the determination of the disturbed electric field around the test body, for example, the rainwater droplet, in its present position, which changes in every time instant and time step, this single solution of the partial differential equation is of a great advantage.

In the present paper, a new proof for the series expansion is given which argues with the line density at the boundary of the test body in two dimensions. So, it does not need any additional, non-physical domain in the neighborhood of the test body. The application of this idea for higher dimensional settings, in particular, for three dimensions, is straightforward if spherical harmonics [17] are used in the Fourier approach.

A mathematical much more challenging topic is the generalization of the series expansion in inhomogeneity indicators for more generally shaped test bodies. For small deviations from the circular form, the ideas in [18] about partial differential equations with perturbated boundaries are a starting point.

\section{References}

[1] G. Joos, Theorectical Physics, Dover, New York, NY, USA, 1987.

[2] P. J. Bailes, J. G. M. Lee, and A. R. Parsons, "An experimental investigation into the motion of a single drop in a pulsed DC electric field," Chemical Engineering Research and Design, vol. 78, no. 3, pp. 499-505, 2000.

[3] K. L. Chrzan, "Concentrated discharges and dry bands on polluted outdoor insulators," in Proceedings of the 13th International Symposium on High Voltage Engineering, J. J. Smit, Ed., Mill Press, Delft, The Netherlands, August 2003.

[4] D. Langemann, "Modelling a droplet moving in an electric field," Mathematics and Computers in Simulation, vol. 68, no. 2, pp. 157-169, 2005.

[5] U. Schreiber and U. van Rienen, "Simulation of the behavior of droplets on polymeric surfaces under the influence of an applied electrical field," in Proceedings of the 9th Biennial IEEE Conference on Electromagnetic Field Computation (CEFC '00), Milwaukee, Wis, USA, June 2000.

[6] U. van Kienen, M. Clemens, and T. Wendland, "Simulation of low-frequency fields on high-voltage insulators with light contaminations," IEEE Transactions on Magnetics, vol. 32, no. 3, pp. 816-819, 1996.

[7] G. Matthies and L. Tobiska, "Simulation of free surfaces of magnetic liquids," in Proceedings of the Algoritmy Conference on Scientific Computing, A. Handlovicova, et al., Ed., pp. 1-10, Bratislava, Slovakia, September 2000. 
[8] C. E. Max, "Strong self-focusing due to the ponderomotive force in plasmas," Physics of Fluids, vol. 19, no. 1 , pp. $74-77,1976$.

[9] D. Langemann, "The total ponderomotive force and inhomogeneity indicators," Mathematical Methods in the Applied Sciences, vol. 30, no. 6, pp. 717-732, 2007.

[10] D. Langemann, "Three-dimensional approximation of the total force on uncharged spheres in electric fields," Journal of Physics A, vol. 40, no. 7, pp. 1687-1701, 2007.

[11] D. Langemann, "Multi-dimensional inhomogeneity indicators and the force on uncharged spheres in electric fields," Mathematical Methods in the Applied Sciences, vol. 32, no. 7, pp. 798-810, 2009.

[12] J. D. Jackson, Classical Electrodynamics, John Wiley \& Sons, New York, NY, USA, 1999.

[13] D. Gilbarg and N. S. Trudinger, Elliptic Partial Differential Equations of Second Order, Classics in Mathematics, Springer, Berlin, Germany, 2001.

[14] V. A. Kozlov, V. G. Mazja, and J. Roßmann, Elliptic Boundary Value Problems in Domains with Point Singularities, vol. 52 of Mathematical Surveys and Monographs, American Mathematical Society, Providence, RI, USA, 1997.

[15] F. G. Tricomi, Integral Equations, Interscience, New York, NY, USA, 1965.

[16] J. S. Walker, Fourier Analysis, Oxford University Press, New York, NY, USA, 1988.

[17] H. Groemer, Geometric Applications of Fourier Series and Spherical Harmonics, vol. 61 of Encyclopedia of Mathematics and Its Applications, Cambridge University Press, Cambridge, UK, 1996.

[18] D. Henry, Perturbation of the Boundary in Boundary-Value Problems of Partial Differential Equations, vol. 318 of London Mathematical Society Lecture Note Series, Cambridge University Press, Cambridge, UK, 2005. 


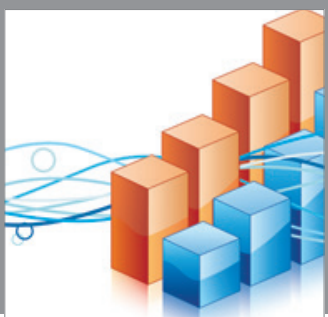

Advances in

Operations Research

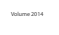

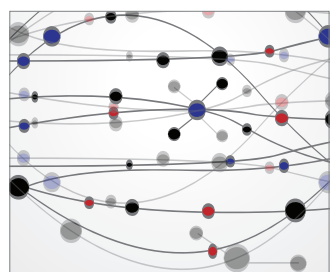

\section{The Scientific} World Journal
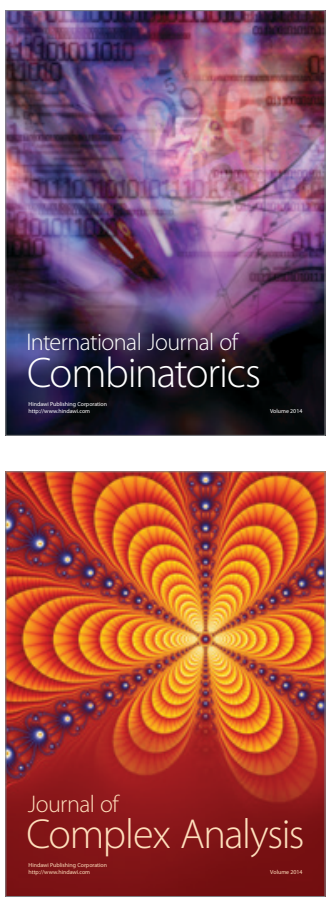

International Journal of

Mathematics and

Mathematical

Sciences
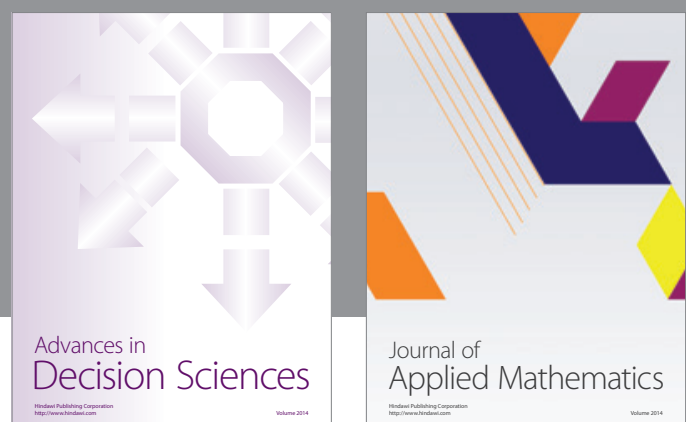

Journal of

Applied Mathematics
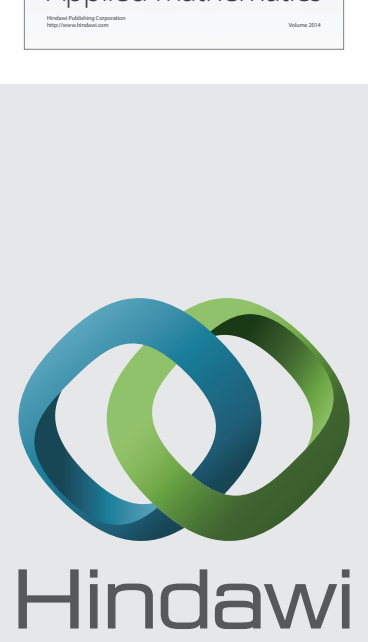

Submit your manuscripts at http://www.hindawi.com
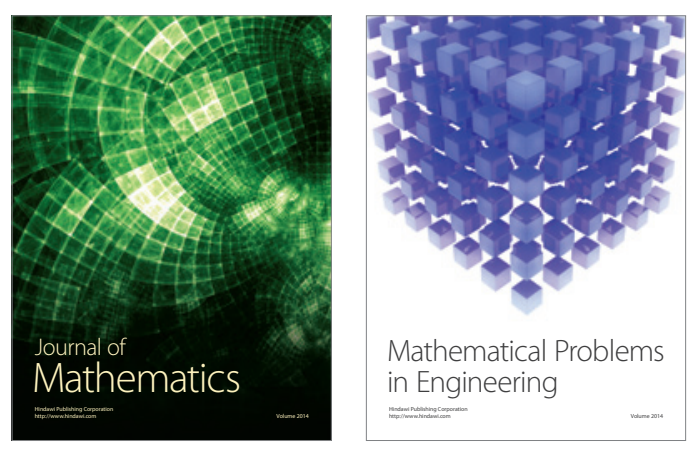

Mathematical Problems in Engineering
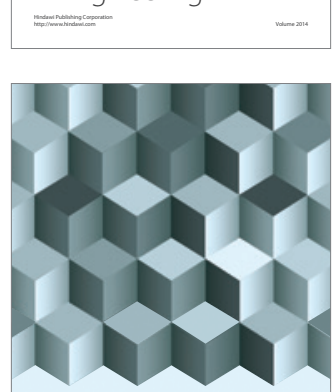

Journal of

Function Spaces
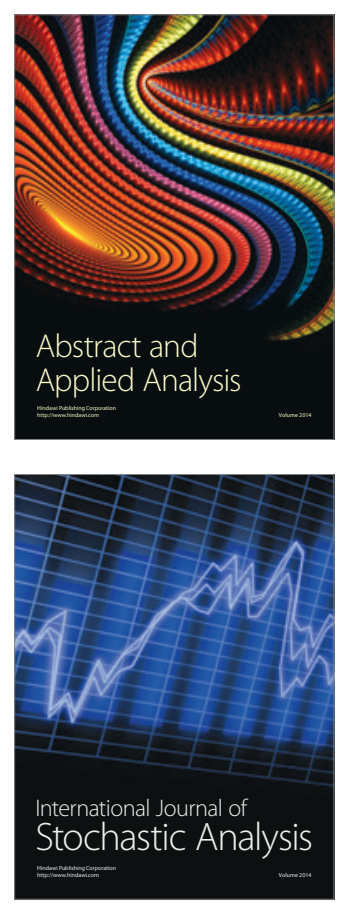

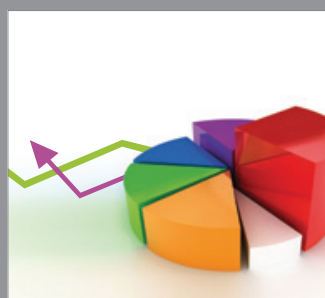

ournal of

Probability and Statistics

Promensencen
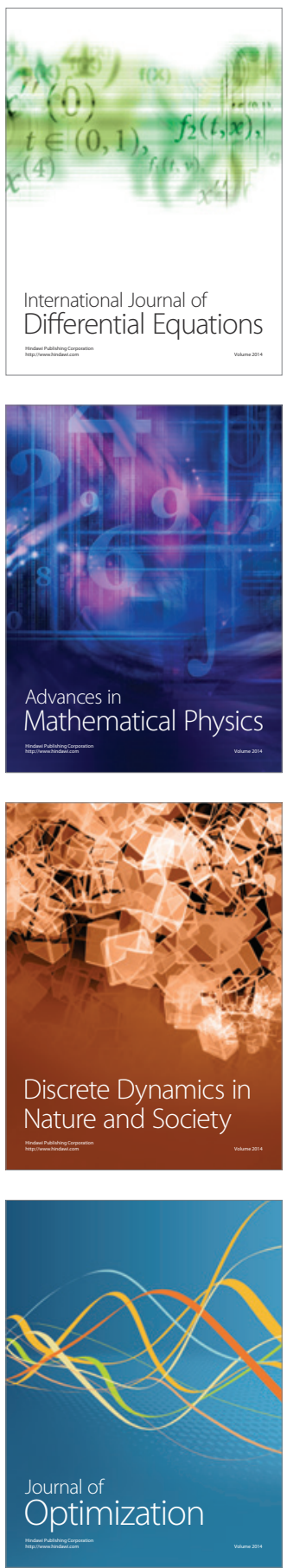